is well-founded, but we believe their subsequent suggestions to be misleading.

Relative asymmetry is the ratio of the absolute difference between the left and right side to the mean trait length. The problem arises here because relative asymmetry is necessarily negatively correlated with its denominator, mean trait length ${ }^{1}$. Use of relative asymmetry will only 'control' for differences in mean trait length when absolute asymmetry is isometrically related to mean trait length. Under such circumstances, relative asymmetry may be a useful index, but isometry must be empirically verified, not assumed. However, belief in the negative correlation between asymmetry and mean trait length does not rest on the dubious use of a ratio variable ${ }^{13}$; Møller and his coworkers $2,4,5,10,11$ ' find the same relationship with absolute asymmetry, as do Sullivan et al. in their analysis of pheasant spurs ${ }^{1}$.

The second criticism hints at a deeper problem in the analysis of asymmetry data, but the suggested solution is inappropriate and the true problem is not addressed. Sullivan et al. note that a shortening of one of a pair of bilaterally symmetrical characters "because of differences in growth rate, injury or damage" both increases absolute asymmetry and reduces mean length, thereby biasing the analysis toward a negative correlation between the two. Their solution is to examine the relationship between absolute asymmetry and the longest of the pair of traits, rather than the mean length. However, this reasoning is flawed on two counts. First, unlike asymmetry through damage or wear, true fluctuating asymmetry (imbalances in symmetrical growth) can result from overgrowth on one side as well as stunting on the other. Thus higher asymmetries through a failure of developmental homeostasis do not inevitably lead to a reduction in the mean trait length, merely an imbalance between the two sides. Second, analysis of the relationship between absolute asymmetry and the longest of the two traits in fact biases one towards finding a positive correlation, as we show below.

The relationship between absolute asymmetry and trait length can be examined in a number of ways. Sullivan et al. consider two: "asymmetry versus mean" and "asymmetry versus longest". However, not all such comparisons are equivalent, or appropriate. In the language of principal component analysis, the mean trait length represents the dimension in which the left and right sides are correlated and absolute asymmetry is the orthogonal dimension in which they are not. However, the dimensions "longest side" and "absolute asymmetry" are not orthogonal,

but positively correlated, thus biasing the analysis. Intuitively, this is most easily understood in the situation where left and right sides are uncorrelated. When the longest side is much greater than the population mean, the short side can assume almost any value (hence asymmetry can be large); but when the longest side approaches the lower limit of the trait size distribution, the shorter side is more constrained (hence asymmetry is low). The problem is greatest when the correlation of left and right sides is relatively loose; precisely the situation that appears typical of display traits $^{4,5,10}$.

In recognizing that damage and wear (but not developmental stress) lead to both an increase in asymmetry and decrease in mean length, Sullivan et al. raise an important point that deserves amplification. As only fluctuating asymmetries, resulting from developmental stress, are of relevance to investigations of the direction and strength of selection, it is vital to exclude any measurements influenced by wear or damage. Sullivan et al. may not be able to distinguish true fluctuating asymmetries from damage or wear in their pheasant spurs, but with sexual plumage, breakages and wear are probably easier to identify a priori. With mean asymmetry

\title{
Predicting Earth's lifespan
}

SIR - Caldeira and Kasting ${ }^{1}$ suggest that Earth's biosphere will exist for more than 1,500 million years, instead of 100 million years as previously suggested $^{2}$. We would like to point out another alternative not considered by these authors.

Caldeira and Kasting did not take into account the $\mathrm{CO}_{2}$ oscillations of recent Earth history ${ }^{3,4}$. The causes of glacialinterglacial oscillations of about 100 p.p.m. are not clear, but they show the possibility of abrupt (on geological timescales) $\mathrm{CO}_{2}$ changes of considerable amplitude. If such $\mathrm{CO}_{2}$ oscillations are a natural behaviour of the global carbon cycle, with the lower mean atmospheric $\mathrm{CO}_{2}$ contents expected in the distant future, very cold climates could ensue. These cold climates might also result if $\mathrm{CO}_{2}$ degassing diminishes with lower geothermal fluxes and slower mantle mixing - an expected consequence of diminishing radioactive decay rates in Earth's mantle ${ }^{5}$.

These factors might lead to a much shorter lifespan for the biosphere because of catastrophic global glaciation, leading to the so-called 'white Earth' solution of energy-balance models ${ }^{6,7}$. The climate of the 'white Earth' can be characterized by very low mean surface temperature (190-240 K) and a thick more sensitive to such outliers than mean trait length, and ornaments perhaps particularly prone to damage, it is all the more important to exclude non-developmental asymmetries from analyses of fluctuating asymmetry. If one can exclude damage and wear effects, an analysis of the relationship between absolute asymmetry and mean trait size is both entirely appropriate and of great theoretical importance ${ }^{10}$.

\section{Innes C. Cuthill}

John P. Swaddle

Mark S. Witter

School of Biological Sciences,

University of Bristol,

Bristol BS8 1UG, UK
1. Sullivan, M. S., Robertson, P. A. \& Aebischer, N. Nature 361, 409-410 (1993).
2. Møller, A. P. Anim. Behav. 40, 1185-1187 (1990)
3. Møiler, A. P. Nature 357, 238-240 (1992).
4. Mølier, A. P. Proc. R. Soc. B248, $199-206$ (1992).
5. Møller, A. P. \& Höglund, J. Proc. R. Soc. B245, 1-5 (1991)
6. Palmer, A. R. \& Strobeck, C. A. Rev. Ecol. Syst. 17 391-421 (1986)
7. Ludwig, W. Das Rechts Links Problem im Tierreich und beim Menschen (Springer, Berlin, 1932)
8. Van Valen. L. Evolution 16, 125-142 (1962)
9. Parsons, P. A. Biol. Rev. 65, 131-145 (1990)
10. Møller, A. P. \& Pomiankowski, A. Genetica (in the press)
11. Møller, A. P. Proc, R. Soc. B243, 59-62 (1991).
2. Manning, J. T. \& Hartley, M. A. Anim. Behav. 42 1020-1021 (1991)
13. Raubenheiner, D. \& Simpson, S. J. Entomol. exp. appl. 62, 221-231 (1992).

$(1,500-3,000 \mathrm{~m})$ layer of ice in the global ocean ${ }^{8}$. This ice sheet would probably not be stable ${ }^{9}$, but such catastrophic glaciation would probably eliminate all higher forms of life. Geothermal and hydrothermal activity would probably continue to support chemotrophic and heterotrophic communities under permanent ice cover.

In conclusion, as far as the 'megabiosphere' is concerned, we concur with Robert Frost's conclusion in 1923 that whereas "some say the world may end in fire, some say ice...that for destruction ice is also great and would suffice".

Andrel Lapenis

Michael R. Rampino

Earth Systems Group,

Department of Applied Science,

New York University,

New York, New York 10003, USA

1. Caldeira, K. \& Kasting, J. Nature $360,721-723$ (1992).

2. Lovelock, J. E. \& Whitfield, M. Nature 296, 561-563 (1982).

3. Lorius, C. et al. Nature 347, 139-145 (1990)

4. Chappellaz, J. et al. Nature 345, 127-131 (1990)

5. Hubbard, W. B. in Planetary Interiors (Van Nostrand Reinhold, New York, 1984).

6. Budyko, M. I. Tellus 21, 611-619 (1969).

7. North, G. R. J. atmos. Sci. 41, 3390-3395 (1975)

8. Verbitsky, M. Ya. \& Chalikov, D. V. Modeling of the Glaciers - Ocean-Atmosphere System (ed. Monin, A. S.) 132 (1986) (in Russian)

9. Caldeira, K. \& Kasting, J. Nature 359, 226-228 (1992) 